\title{
An Overview on Opinion Spreading Model
}

\author{
Pei Liu, Xi Chen \\ School of Automation, Huazhong University of Science and Technology, Wuhan, China \\ Email: ouch208@163.com
}

Received February 2015

\begin{abstract}
Research on opinion spreading has received more and more attention in recent years. This paper focus on make a summary of opinion evolution researches, we first review some classical opinion models, and then introduce the existing result of improvement models from the aspect of opinion space, model parameter, social network and so on. The current study's limitation and further research are also prospected at the end. By in-depth understand the opinion spreading mechanism so as to guide and control the public opinions, which is very useful and meaningful.
\end{abstract}

\section{Keywords}

\section{Opinion Spreading, Classical Model, Spreading Mechanism}

\section{Introduction}

In recent years, many researchers have made significant contributions to the field of opinion evolution. Public opinion makes a great difference to politics and economics and so on. The behavior of individual in the society are susceptible to the influence of public opinion, triggered by public opinion of social events often occur. Thus in order to prevent the disaster, and control the spreading of negative public opinion, we need to understand the formation and propagation mechanism of public opinion. Each individual always hold his attitude or opinion for any event, while his opinion will be changed after communicate with his neighbors. In this paper, we make an overview summary on opinion spreading dynamics, from the classical model to improved models, focus on model parameters, the communication mechanism, the network topology to analysis the existing results. We will introduces the classical opinion evolution models in the next section, and in Section 3, we will introduce the further study of opinion spreading models from the five main aspect.

\section{Classical Models}

In recent years, agent-based computer simulations are viewed as an innovative way of simulating many social behaviors grounded on minimal rules, simplicity and emergence. Most opinion evolution researches using agentbased computer simulations. In the existing study of opinion spreading, the models can be divided into discrete opinion model and continuous models. The most popular continuous opinion spreading models consists of Deffuant model [1] and Hegselmann-Krause model [2]; while the discrete models including Sznajd model [3], voter model [4], Galam majority rule model [5] and social impact model [6]. We will simply introduce the evolving rules of these models in the following. 


\subsection{Deffuant Mode}

There are $N$ agents in the system, each agents holds a continuous opinion. In $x_{i}(t)$ each time step, two agents are random chose, if their opinion distance below the opinion threshold $\left|x_{i}(t)-x_{j}(t)\right|<d$, they can communicate according to the following rules, otherwise, they keep their opinion unchanged.

$$
\left\{\begin{array}{l}
x_{i}(t+1)=x_{i}(t)+\mu\left(x_{j}(t)-x_{i}(t)\right) \\
x_{j}(t+1)=x_{j}(t)+\mu\left(x_{i}(t)-x_{j}(t)\right)
\end{array}\right.
$$

The opinion convergence parameter $\mu$ values from 0 to 0.5 .

\subsection{HK Model}

There are $N$ agents in the system, each agents holds an continuous opinion $x_{i}(t)$. The same with Deffuant model, each individual choose communicate neighbors according to the bounded confidence principle, they only communicate with neighbors who share the similar opinion, namely $I(i, x)=\left\{1 \leq j \leq n|| x_{i}-x_{j} \mid \leq \varepsilon_{i}\right\}$. At each time step, the agents change their opinion on the basis of the following rules:

$$
x_{i}(t+1)=|I(i, x(t))|^{-1} \sum_{j \in I(i, x(t))} x_{j}(t), \quad t \in T
$$

\subsection{Sznajd Model}

The system is composed of $N$ agents, each agents can only hold opinions of two choice, $s_{i}=A$ or $\mathrm{B}$. The model based on the thesis of "three people spreading reports of a tiger make you believe there is one around". At each time step, random select an agent $i$ and agent $i+1$ to influence their nearest neighbors' opinion, agent $i-1$ and agent $i+1$. Dynamic rules as follows:

1) If $s_{i}=s_{i+1}$, then they will affect their neighbors agent $i-1$ and $i+1, s_{i-1}=s_{i}=s_{i+1}=s_{i+2}$;

2) If $s_{i} \neq s_{i+1}$, then they can only affect each other's neighbor, $s_{i-1}=s_{i+1}, s_{i}=s_{i+2}$.

\subsection{Voter Model}

$\mathrm{N}$ agents, each agent has opinion 0 or 1 in the system. The model is base on Ising model, and processes a very simple dynamic rule. In every step, first choose an agent at random, and then the agent will random adopt one of his neighbor's opinion as his own attitude.

\subsection{Galam Majority Rule Model}

There are $N$ agents in the system, among them, the proportion of agents with opinion $s_{i}=1$ is $p_{+}$, while the other $p_{-}=1-p_{+}$agent hold the opinion $s_{i}=-1$. The model take fully connected network into consideration, namely every agent can communicate with all the other agents in the network. At each time step, first find $r$ agents at random and regard them as a community, second find out the majority opinion of the community, at last make everyone in the community replace their opinion with the majority opinion.

\subsection{Social Impact Model}

This model consider the social impact is the main factor that affecting the opinion change, while the social impact including interactive, reciprocal, and recursive operation. In summary, there are two mian factor to decide agents' opinion, recursive impact $\hat{I}_{p}$ and supportive impact $\hat{I}_{s}$. For an individual, the supportive impact refers to the neighbors' impact who share the same opinion, while the oppose opinion neighbors in the social will have recursive impact on the individual. The calculation formula, respectively:

$$
\begin{gathered}
\hat{I}_{p}=N_{0}^{1 / 2}\left[\sum\left(p_{i} / d_{i}^{2}\right) / N_{0}\right] \\
\hat{I}_{s}=N_{s}^{1 / 2}\left[\sum\left(s_{i} / d_{i}^{2}\right) / N_{s}\right]
\end{gathered}
$$

$N_{0}$ and $N_{s}$ respectively refers to the number of individuals with an opposing view and individuals sharing 
the individual's view (including the individual), $p_{i}$ and $s_{i}$ denotes the persuasiveness of individual $i$ with an opposing view or the same view, $d_{i}$ the distance between individual $i$ and the recipient. At each time step, for each agent, get his recursive impact $\hat{I}_{p}$ and supportive impact $\hat{I}_{s}$. If $\hat{I}_{p} / \hat{I}_{s}>1$, then he will change his opinion based on his past opinion, and random generate the new recursive impact $\hat{I}_{p}$ and supportive impact $\hat{I}_{s}$, otherwise, he won't change his opinion.

According to the above classical opinion models, no matter spreading on probability or some rules of use neighbors' opinion, they are all concentrate on opinion convergence when reach stable state. After the emergence of classical models, many further researches focus on the models, from the model parameter, social network and evolution mechanism.

\section{Further Study on Classical Model}

\subsection{Opinion Space}

Classical models can be divide into discrete and continuous models according to opinion, while in the latter study, discrete opinion also be introduced into continuous models such as KH model and Deffuant model, the result show that compared with continuous opinion, the discrete opinion make the model algorithms faster and more stable [7]-[9].

On the other hand, use a simple value to express one's opinion will ignore one's subjective willingness. Thus some studies introduce the relative agreement opinion model. The model adding uncertainly which decide the intensity of influence on one's opinion. Two agents' opinion overlap minus non-overlap part means their agreement. The communication does not only change opinion but also uncertainly [10] [11].

The prime model all assume that the system exist only one subject and each agents only hold one opinion, however, agents have opinions on different subjects. Thus vector are introduced to show the opinion since agents may have opinions on dierent subjects, the simulation result tells there mostly exists no absolute consensus in multidimensional opinion model [9] [12].

\subsection{Model Parameters}

Confidence bounded-whether in Deffaunt model or in $\mathrm{KH}$ model, the confidence bounded are the main factors that influencing the opinion convergence and driven stabilization. There is a critical threshold of confidence bounded in continuous models, when above the value all opinion can reach a consensus state, and the threshold remain to be 0.5 unaffected by network topology. On the contrary when below the threshold the whole opinion is difficult to reach agreement [13]-[16]. On the other hand, everyone share the same confidence bounded that is clearly not conform to the reality. Some study suggest that everyone should hold different confidence bounded, they let confidence dounded of Deffaunt model follow a random distribution or power law distribution, the result show that the opinion will reach consensus if expectations of confidence bounded beyond 0.5, and the confidence bounded distribution also affect the convergence speed, sharply distribution converge faster than broadly distribution [17] [18].

First impression-The initial opinion distribution or first impression also attract attentions, in classic models, the first impression always follows uniform distribution, some study make it follows different random distribution, and proposed that the confidence bounded critical threshold which is related to initial opinion distribution, and the different first impression distribution makes consensus time different [9] [19]. what's more, the Deffuant algorithm is mainly average the initial input [20].

Interaction parameter-also call convergence parameter $\mu$, used to express the influence degree of communication neighbors on individual's own opinion in Deffuant model By investigate homogeneous and heterogeneous converse parameter, the result show that the parameter only influence the opinion consensus time, but have nothing to do with final opinion distribution [16].

\subsection{Network Topology}

In the beginning, those models often studied in a square lattice thus everyone can only communicate with his four neighbors, or some study apply in the fully-connected network thus everyone can communicate with all other person. Since complex network become more and more popular, more and more study concentrate on modeling opinion evolution on complex social network, such as random networks [21], scale-free networks [7] 
and small-world networks [22], to make the model more realistic. Finding that network topology greatly influenced the opinion consensus, scale-free network can speed up opinion evolution compared with small-world network [16].

In order to further study the topology of the network, many scholars consider the directed network. Gandica compared opinion evolution on directed and undirected small-word networks, it is more difficult for directed WS network to reach consensus because it have less links. On the other hand, the network size have no impact on opinion final state, while confidence bounded have great influence than small world rewiring probability [23]. And the researches about direct and undirected scale-free network on opinion spreading models are also in progress [24] [25]. In short, the directed network has the same influence on KH model, while have different effects and get different result on Deffuant model [26].

\subsection{Social Diversity}

After in-depth study of classical model, although adding network topology makes the opinion evolution model more realistic, the simple opinion spreading or exchange mechanism still bounded by the assumption. Therefore, a lot of researches are put forward according to the basic model of different improvement.

The classical network consider all individuals the same, and ignoring the social diversity. While in real life, individual's character will be affect by social environment, thus his spreading rules will be changed too. Various modifications of the classical models aimed at making the model to be more realistic to show some real phenomenon. Introduce the social power into opinion spreading model is one of the most notable achievements to reflect the social diversity. Social power refers to that each individual have different influence on other individuals. In social network, mainly use an average degree of the node to represent his social power. The result show that the presence of hub nodes make heterogeneous scale-free network easier to reach consensus by introduce the social diversity in this definition [27] [28].

There are some studies divide agents into two or three types base on agent attribute parameters. Such as social power, node degree, betweenness, vulnerability and so on are used to evaluate the social power, and leader have stronger impact (social power) on neighbors than followers [21] [29]. Some studies introduce a "confidence" parameter or self-belief based on convergence parameter to weighting how much the agent trust his own opinion with respect to those of others, and classified agents into three categories according to different self-belief. Open-minded agents or followers have a low self-belief and therefore are more susceptible to neighbors, closedminded or leaders agents have a high self-belief, and moderate-minded agents [30] [31]. This mechanism has also been applied to other model [32] [33]. The result show that agents with different self-belief level have different impact on opinion spreading, closed-minded agents have negative effect on opinion consensus, while the open-minded agents can't speed up convergence as expect.

Communication rules also can be used to performance the social diversity, individuals can be divided into two categories-leader and follower, or informed agents and major agents, etc. They comply with different rules, during the communication the leaders won't change their opinion or they opinion only can be affect by leaders, while the follower obey the same rule as classical models, both leaders and followers will influence their opinion. In such system, the leader has a great influence on opinion evolution no matter in which network topology [11] [34]-[36].

In either classification methods, leadership nodes are have a great impact on the opinion spreading, we can control the whole network opinion to reach consensus by leaders.

\subsection{Improvement on Spreading Mechanism}

In order to reproduce the most common phenomenon of real life, the classical model always ignore some special cases, make the communication condition too harsh. For example, the models assume that only when agents share the similar opinion will exchange opinion, while in real life, individuals also spreading opinions to distinct neighbors. Many researches improved the model by considerate such special conditions, such as add similarity-based random neighbors into communicate neighborhood, outside the confidence bounded two agents also can change opinion based on random selection, the model will always reaches consensus [37]. The long-range multi-choice (LMDW) model aim at study the influence of this special conditions on opinion spreading, and Grauwin consider it as an interaction noise [38] [39]. On the contrary, others think communication conditions of the classical model are too broad because the communication neighbors only be chose by opinion similarity, 
while there are some more factors to exclude communication neighbors. Such as eyeshot limit mechanism, that is to say, beside opinion similarity, individuals can only communicate with neighbors within limited geography distance [40]. Adding bounded influence parameter is another way to filter the neighbors [17].

\section{Conclusions}

Researches on opinion spreading or evolution models have received a lot of achievement in recent years, but still need to be further studies. Current models mostly based on discrete or continuous opinion value to represent individuals' attitude and behavior, thus the complexities of individuals are ignored, and sometimes they won't perform the same as they think. This kind of study also has been put forward recently, such as continuous opinion and discrete action models (CODA models). In addition, sociology or complex network theory can be related to opinion spreading mechanism to make the model more realistic, such as individual diversity. Since the Internet become an essential part of our life, it is important to study the influence of online network on opinion spreading.

Everyone lives in the society, influenced by the environment, public opinion will affect individuals around the thinking and behavior. Especially when deal with the emergency, understand the opinion spreading mechanism, further to predict and control the public opinions, will be helpful for social stability. Therefore Study of the evolution of public opinion has great significance and application value.

\section{Acknowledgements}

This work was supported by the National Natural Science Foundation of China (Grant No. NSFC 70903026) and the Fundamental Research Funds for the Central Universities (HUST: 2013TS125).

\section{References}

[1] Deffuant, G., et al. (2000) Mixing Beliefs among Interacting Agents. Advances in Complex Systems, 3, 87-98.

[2] Hegselmann, R. and Krause, U. (2002) Opinion Dynamics and Bounded Confidence Models, Analysis, and Simulation. Journal of Artificial Societies and Social Simulation, 5.

[3] Sznajd-Weron, K. and Sznajd, J. (2000) Opinion Evolution in Closed Community. International Journal of Modern Physics C, 11, 1157-1165. http://dx.doi.org/10.1142/S0129183100000936

[4] Clifford, P. and Sudbury, A. (1973) A Model for Spatial Conflict. Biometrika, 60, 581-588. http://dx.doi.org/10.1093/biomet/60.3.581

[5] Galam, S. (2002) Minority Opinion Spreading in Random Geometry. The European Physical Journal B-Condensed Matter and Complex Systems, 25, 403-406. http://dx.doi.org/10.1140/epjb/e20020045

[6] Nowak, A., Szamrej, J. and Latané, B. (1990) From Private Attitude to Public Opinion: A Dynamic Theory of Social Impact. Psychological Review, 97, 362. http://dx.doi.org/10.1037/0033-295X.97.3.362

[7] Stauffer, D., Sousa, A. and Schulz, C. (2004) Discretized Opinion Dynamics of the Deffuant Model on Scale-Free Networks. Journal of Artificial Societies and Social Simulation, 7.

[8] Fortunato, S. (2004) The Krause-Hegselmann Consensus Model with Discrete Opinions. International Journal of Modern Physics C, 15, 1021-1029. http://dx.doi.org/10.1142/S0129183104006479

[9] Shang, Y.L. (2013) Deffuant Model with General Opinion Distributions: First Impression and Critical Confidence Bound. Complexity, 19, 38-49. http://dx.doi.org/10.1002/cplx.21465

[10] Amblard, F. and Deffuant, G. (2004) The Role of Network Topology on Extremism Propagation with the Relative Agreement Opinion Dynamics. Physica A: Statistical Mechanics and Its Applications, 343, 725-738.

[11] Kurmyshev, E., Juárez, H.A. and González-Silva, R.A. (2011) Dynamics of Bounded Confidence Opinion in Heterogeneous Social Networks: Concord against Partial Antagonism. Physica A: Statistical Mechanics and Its Applications, 390, 2945-2955.

[12] Jacobmeier, D. (2005) Multidimensional Consensus Model on a Barabási-Albert Network. International Journal of Modern Physics C, 16, 633-646. http://dx.doi.org/10.1142/S0129183105007388

[13] Fortunato, S. (2004) Universality of the Threshold for Complete Consensus for the Opinion Dynamics of Deffuant et al. International Journal of Modern Physics C, 15, 1301-1307. http://dx.doi.org/10.1142/S0129183104006728

[14] Fortunato, S. (2005) On the Consensus Threshold for the Opinion Dynamics of Krause-Hegselmann. International Journal of Modern Physics C, 16, 259-270. http://dx.doi.org/10.1142/S0129183105007078 
[15] Lorenz, J. (2005) A Stabilization Theorem for Dynamics of Continuous Opinions. Physica A: Statistical Mechanics and Its Applications, 355, 217-223.

[16] Zhang, W., He, M.-S. and Jin, R. (2014) Opinion Dynamics in Complex Networks. Lithuanian Journal of Physics, 53.

[17] Liang, H.L., Yang, Y.P. and Wang, X.F. (2013) Opinion Dynamics in Networks with Heterogeneous Confidence and Influence. Physica A, 392, 2248-2256. http://dx.doi.org/10.1016/j.physa.2013.01.008

[18] Shang, Y.L. (2014) An Agent Based Model for Opinion Dynamics with Random Confidence Threshold. Communications in Nonlinear Science and Numerical Simulation, 19, 3766-3777. http://dx.doi.org/10.1016/j.cnsns.2014.03.033

[19] Lorenz, J. and Urbig, D. (2007) About the Power to Enforce and Prevent Consensus by Manipulating Communication Rules. Advances in Complex Systems, 10, 251-269. http://dx.doi.org/10.1142/S0219525907000982

[20] Jacobmeier, D. (2006) Focusing of Opinions in the Deffuant Model: First Impression Counts. International Journal of Modern Physics C, 17, 1801-1808. http://dx.doi.org/10.1142/S0129183106010108

[21] Jalili, M. (2013) Social Power and Opinion Formation in Complex Networks. Physica A: Statistical Mechanics and Its Applications, 392, 959-966.

[22] Li, P.-P., Zheng, D.-F. and Hui, P.M. (2006) Dynamics of Opinion Formation in a Small-World Network. Physical Review E, 73, Article ID: 056128. http://dx.doi.org/10.1103/PhysRevE.73.056128

[23] Gandica, Y., et al. (2010) Continuous Opinion Model in Small-World Directed Networks. Physica A: Statistical Mechanics and Its Applications, 389, 5864-5870.

[24] Stauffer, D. and Meyer-Ortmanns, H. (2004) Simulation of Consensus Model of Deffuant et al. on a Barabasi-Albert Network. International Journal of Modern Physics C, 15, 241-246. http://dx.doi.org/10.1142/S0129183104005644

[25] Jacobmeier, D. (2005) Multidimensional Consensus Model on a Barabási-Albert Network. International Journal of Modern Physics C, 16, 633-646. http://dx.doi.org/10.1142/S0129183105007388

[26] Stauffer, D. (2005) Sociophysics Simulations II: Opinion Dynamics. arXiv preprint physics/0503115

[27] Jalili, M. (2013) Social Power and Opinion Formation in Complex Networks. Physica A: Statistical Mechanics and Its Applications, 392, 959-966.

[28] Jalili, M. (2013) Effects of Leaders and Social Power on Opinion Formation in Complex Networks. Simulation, 89, 578-588. http://dx.doi.org/10.1177/0037549712462621

[29] Boccara, N. (2008) Models of Opinion Formation: Influence of Opinion Leaders. International Journal of Modern Physics C, 19, 93-109. http://dx.doi.org/10.1142/S0129183108011954

[30] Weisbuch, G., et al. (2002) Meet, Discuss, and Segregate! Complexity, 7, 55-63. http://dx.doi.org/10.1002/cplx.10031

[31] Hou, L., et al. (2014) Prediction of Collective Opinion in Consensus Formation. International Journal of Modern Physics C, 25.

[32] Ramirez-Cano, D. and Pitt, J. (2006) Follow the Leader: Profiling Agents in an Opinion Formation Model of Dynamic Confidence and Individual Mind-Sets. IEEE/WIC/ACM International Conference on Intelligent Agent Technology, IAT'06.

[33] Fu, G.Y., Zhang, W.D. and Li, Z.J. (2015) Opinion Dynamics of Modified Hegselmann-Krause Model in a GroupBased Population with Heterogeneous Bounded Confidence. Physica A: Statistical Mechanics and Its Applications, 419, 558-565.

[34] Jalili, M. (2013) Effects of Leaders and Social Power on Opinion Formation in Complex Networks. Simulation, 89, 578-588. http://dx.doi.org/10.1177/0037549712462621

[35] Afshar, M. and Asadpour, M. (2010) Opinion Formation by Informed Agents. Journal of Artificial Societies and Social Simulation, 13, 5.

[36] Borra, D. and Lorenzi, T. (2013) A Hybrid Model for Opinion Formation. Zeitschrift für Angewandte Mathematik und Physik, 64, 419-437. http://dx.doi.org/10.1007/s00033-012-0259-z

[37] Liu, Q.P. and Wang, X.F. (2013) Opinion Dynamics with Similarity-Based Random Neighbors. Scientific Reports, 3.

[38] Grauwin, S. and Jensen, P. (2012) Opinion Group Formation and Dynamics: Structures That Last from Nonlasting Entities. Physical Review E, 85, Article ID: 066113. http://dx.doi.org/10.1103/PhysRevE.85.066113

[39] Zhang, J.B. and Hong, Y.G. (2013) Opinion Evolution Analysis for Short-Range and Long-Range Deffuant-Weisbuch Models. Physica A, 392, 5289-5297. http://dx.doi.org/10.1016/j.physa.2013.07.014

[40] Li, S.S. and Zhang, S.Y. (2010) Leader and Follower: Agents in an Opinion Dynamics and Bounded Confidence Model on the Stochastic Movement World. IEEE 2nd International Conference on Computational Intelligence and Natural Computing Proceedings (CINC). 